\title{
Expression of midkine and vascular endothelial growth factor in gastric cancer and the association of high levels with poor prognosis and survival
}

\author{
ZHI-QIANG ZHAO ${ }^{1 *}$, SHENG YANG ${ }^{2 *}$ and HUI-SHAN LU ${ }^{2}$ \\ ${ }^{1}$ Department of Oncology, The First Hospital of Putian, Putian 351100; ${ }^{2}$ Department of Oncology, \\ the Union Hospital of Fu Jian Medical University, Fuzhou 350001, P.R. China
}

Received July 25, 2011; Accepted October 14, 2011

DOI: $10.3892 / \mathrm{mmr} .2011 .649$

\begin{abstract}
The aim of the present study was to investigate the expression of midkine (MK) and vascular endothelial growth factor (VEGF) in gastric cancer and its relationship with gastric cancer prognosis and survival rate. We recruited 107 patients with complete clinical data and available tissue samples [gastric cancer tissue ( $\mathrm{n}=107)$; adjacent normal gastric mucosa $(n=31)]$. MK and VEGF expression in these tissues were assayed by immunohistochemistry. The association of MK or VEGF expression with various prognostic factors in gastric cancer and the 5-year survival of gastric cancer patients were analyzed. MK and VEGF immunoreactivity were detected in $69.2 \%$ (74 out of 107 cases) and $66.4 \%$ ( 71 out of 107 cases) of gastric cancer tissues, but not in normal gastric tissues $(\mathrm{P}=0.00)$. MK and VEGF expression was correlated with tumor size, depth of invasion, lymph node metastasis and pathological stage $(\mathrm{P}<0.01)$, but not with age and gender $(\mathrm{P}>0.05)$. MK expression was positively correlated with VEGF expression $(r=0.681, \mathrm{P}<0.01)$. In addition, MK or VEGF expression was negatively correlated with the 5 -year survival rate $(\mathrm{P}<0.01)$. The 5 -year survival rate was significantly higher in patients with MK- or VEGF-immunonegative tumors than in patients with immunopositive ones $(\mathrm{P}<0.01)$. Co-expression of MK and VEGF was an independent predictor of gastric cancer prognosis. Expression of MK and VEGF is increased in gastric cancer and increased expression is closely correlated with poor prognosis and survival.
\end{abstract}

Correspondence to: Dr Hui-Shan Lu, Department of Oncology, The Union Hospital of Fu Jian Medical University, Fuzhou 350001, P.R. China

E-mail:1hs0591@sina.cn

${ }^{*}$ Contributed equally

Key words: gastric cancer, vascular endothelial growth factor, midkine, immunohistochemistry, prognosis

\section{Introduction}

Gastric cancer is a common malignancy globally and remains a disease of high morbidity and mortality (1-3). Tumor development is an extremely complex biological process involving multiple genes, factors and stages. In some sense, cancer is a genetic disease. Therefore, understanding the molecular genetics of gastric cancer may reveal new strategies for the prevention and treatment of gastric cancer in the future (4-6). Angiogenesis factors and various cytokines appear to be involved in tumor cell proliferation, invasion and metastasis. Midkine (MK) is a recently discovered heparin-binding growth factor with strong differentiation, mitotic and angiogenesis activity (7-9). Vascular endothelial growth factor (VEGF) promotes vascular endothelial cell proliferation and its high expression in tumors is significantly associated with advanced disease and poor prognosis (10-12). We investigated MK and VEGF expression levels in gastric cancer specimens by immunohistochemistry, and examined their relationship to prognostic factors of gastric cancer.

\section{Materials and methods}

Patients and tissue specimens. A total of 107 patients (80 males, 27 females; age $>60$ years, $n=52 ; \leq 60$ years, $n=55$ ), who received surgery for gastric adenocarcinoma at the Department of Oncology, Union Hospital of Fujian Medical University in 2002, were randomly selected. Complete clinical follow-up data and paraffin-embedded gastric cancer specimens were available for all patients. In addition, paraffin-embedded normal gastric mucosa specimens from 31 patients treated during the same period served as controls. Gastric cancer patients were staged using the International Union Against Cancer (UICC) 1997 TNM staging criteria, and histological typing of the primary tumor was performed using the World Health Organization (WHO) criteria. Undifferentiated $(n=7)$, poorly differentiated $(n=33)$, well differentiated $(n=32)$ and moderately differentiated $(n=35)$ gastric adenocarcinoma, either with $(n=88)$ or without lymph node metastasis $(\mathrm{n}=19)$, and either depth of invasion $\mathrm{pT}_{1}$ $+\mathrm{pT}_{2}(\mathrm{n}=23)$ or $\mathrm{pT}_{3}+\mathrm{pT}_{4}(\mathrm{n}=84)$, was diagnosed. There were 30 patients in TNM stage I+ II and 77 in TNM stage III + IV.Prior to surgery, no patients received radiotherapy or chemotherapy. 
Table I. Correlation of MK or VEGF expression with clinicopathological characteristics.

\begin{tabular}{|c|c|c|c|c|c|c|c|c|c|}
\hline \multirow{2}{*}{$\begin{array}{l}\text { Clinicopathological } \\
\text { characteristics }\end{array}$} & \multirow{2}{*}{$\begin{array}{l}\text { Case } \\
\text { (no.) }\end{array}$} & \multicolumn{4}{|c|}{ MK } & \multicolumn{4}{|c|}{ VEGF-A } \\
\hline & & + & - & $\chi^{2}$ test & P-value & + & - & $\chi^{2}$ test & P-value \\
\hline \multicolumn{10}{|l|}{ Age (years) } \\
\hline$>60$ & 52 & 35 & 17 & 0.163 & 0.687 & 37 & 15 & 1.043 & 0.307 \\
\hline$\leq 60$ & 55 & 34 & 21 & & & 34 & 21 & & \\
\hline \multicolumn{10}{|l|}{ Gender } \\
\hline Male & 80 & 55 & 25 & 0.025 & 0.875 & 51 & 29 & 0.964 & 0.326 \\
\hline Female & 27 & 19 & 8 & & & 20 & 7 & & \\
\hline \multicolumn{10}{|c|}{ Tumor size (diameter, $\mathrm{cm}$ ) } \\
\hline$>5$ & 49 & 43 & 6 & 14.656 & 0.000 & 42 & 7 & 15.175 & 0.000 \\
\hline$\leq 5$ & 58 & 31 & 27 & & & 29 & 29 & & \\
\hline \multicolumn{10}{|l|}{ Depth of invasion } \\
\hline $\mathrm{T} 1+\mathrm{T} 2$ & 23 & 5 & 18 & 30.887 & 0.000 & 4 & 19 & 31.462 & 0.000 \\
\hline $\mathrm{T} 3+\mathrm{T} 4$ & 84 & 69 & 15 & & & 67 & 17 & & \\
\hline \multicolumn{10}{|c|}{ Degree of differentiation } \\
\hline \multicolumn{10}{|c|}{ Lymph node metastasis } \\
\hline No & 19 & 2 & 17 & 37.235 & 0.000 & 3 & 16 & 32.499 & 0.000 \\
\hline Yes & 88 & 72 & 16 & & & 72 & 16 & & \\
\hline \multicolumn{10}{|l|}{ TNM stages } \\
\hline $\mathrm{I}+\mathrm{II}$ & 30 & 6 & 24 & 47.232 & 0.000 & 5 & 25 & 46.103 & 0.000 \\
\hline $\mathrm{III}+\mathrm{IV}$ & 77 & 68 & 9 & & & 66 & 11 & & \\
\hline
\end{tabular}

PTNM, pathological tumor node metastasis; MK, midkine; VEGF, vascular endothelial growth factor.

Patients were followed up post-operatively in the hospital or by home visits, letters and telephone calls. The follow-up rate was $100 \%$ and the follow-up period was 72 months (after excluding those who succumbed during follow-up). Survival was defined as the time elapsed between initial surgery and the day of the last follow-up, mortality or loss of contact.

Immunohistochemical method. Tissues were fixed with $4 \%$ formaldehyde and paraffin-embedded. Serial 5- $\mu \mathrm{m}$ sections were affixed to a number of poly-resistant acid coated glass slides, placed in a $70^{\circ} \mathrm{C}$ oven for $4 \mathrm{~h}$ to dry, stained with VEGF monoclonal antibody using an SP immunohistochemistry kit and DAB color reagent all purchased from Fuzhou Maixin Bio-Tech. Co. (Fujian, China), and stained with MK monoclonal antibodies according to the manufacturer's instructions (SantaCruz Biotechnology, Santa Cruz, CA, USA). Phosphate-buffered saline (PBS) instead of primary antibody was used as the negative control. The positive control was provided by the company.

Evaluation of the staining reaction. Brown or brown-yellow staining signals found in the cell membrane or cytoplasm were considered to indicate MK and VEGF immunopositivity. The negative controls were unstained. The number of positive cells in 500 tumor cells was counted within 5 randomly selected high power fields (x400). Four grades were defined according to the percentage of positively stained cells: 0 , no immunopositive cells; $1,<25 \%$ immunopositive cells; $2,25-50 \%$ immunopositive cells; $3,>50 \%$ immunopositive cells. Four
Table II. Correlation of MK and VEGF expression in gastric cancer.

\begin{tabular}{lrrrrr}
\hline & \multicolumn{2}{c}{ VEGF } & & & \\
\cline { 2 - 3 } MK & - & + to ++ & Total & $\chi^{2}$ test & P-value \\
\hline- & 27 & 6 & 33 & & \\
+ to ++ & 9 & 65 & 74 & 46.529 & 0.00 \\
Total & 36 & 71 & 107 & & \\
\hline
\end{tabular}

MK, midkine; VEGF, vascular endothelial growth factor.

grades were defined according to color-staining intensity: 0 , no color; 1 , weak, pale yellow; 2 , medium, brown; 3 , strong, dark brown. The final overall score (determined by summing the two grades) was: 0-2, negative, '-'; 3-4, weakly positive, '十'; and 5-6, strongly positive, '++'. Overall scores of ' + ' and ' ++ ' were all considered as positive to simplify data processing.

Statistical analysis. All data were analyzed using SPSS v.11.5. Categorical variables were compared using the $\chi^{2}$ test and continuous variables were compared using a t-test. The correlation between two variables was evaluated using Spearman rank correlation analysis. $\mathrm{P}<0.05$ was considered to be statistically significant. Survival curves were drawn using the Kaplan-Meier method, and differences between the curves 


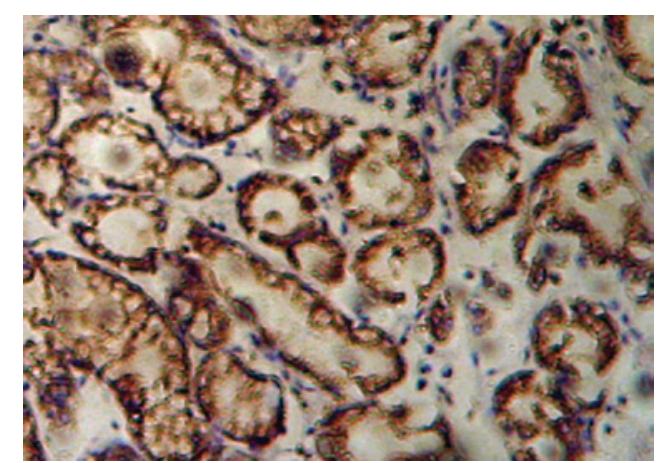

Figure 1. Positive expression of MK in gastric carcinoma tissue (SP x200).

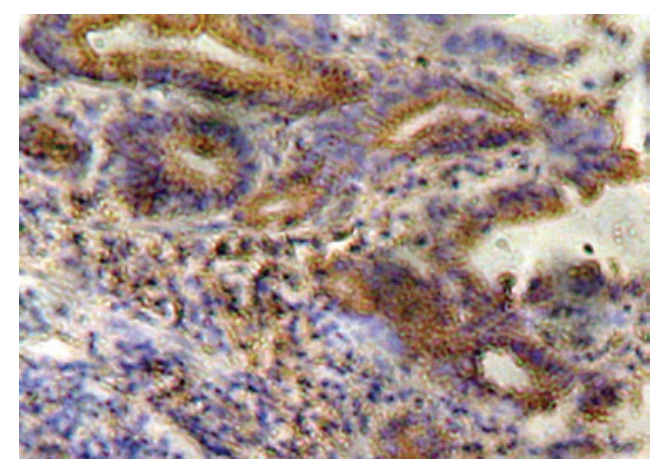

Figure 2. Positive expression of VEGF-A in gastric carcinoma tissue (SP x200).

were tested using the log-rank test. To identify the prognostic factors of gastric cancer, a Cox regression model of multivariate analysis was used.

\section{Results}

MK and VEGF expression in gastric cancer tissues. MK and VEGF were highly expressed in 69.2 and $66.4 \%$, respectively, of gastric cancer specimens, mainly in the tumor cell cytoplasm. Immunopositive cells were widely distributed in cancer tissues, particularly in and around vascular endothelium. As shown in Figs. 1 and 2, no MK and VEGF was expressed in normal gastric mucosa. A statistically significant difference in MK and VEGF expression was found between tumor and control tissue specimens $(\mathrm{P}=0.00)$. The expression of $\mathrm{MK}$ and VEGF was positively correlated with tumor size, depth of invasion, differentiation, lymph node metastasis and pathological staging $(\mathrm{P}<0.05)$ (Table I).

MK expression in gastric cancer was associated with VEGF expression. Among 74 specimens immunopositive for $\mathrm{MK}$, 65 were also immunopositive for VEGF (87.8\%). In 33 tissues immunonegative for MK, 6 were immunopositive for VEGF (18.2\%). The rate of $\mathrm{MK}^{+} \mathrm{VEGF}^{+}$expression was significantly different from the rate of $\mathrm{MK}^{-} \mathrm{VEGF}^{+}$expression $(\mathrm{P}<0.01)$. Additionally, VEGF expression in gastric cancer was positively correlated with MK expression $(\mathrm{r}=0.681, \mathrm{P}<0.01)$ (Table II).

$M K$ and VEGF expression was correlated with poor survival. The 5-year survival rate of patients with MK-and VEGF-positive tumors was 25.41 and $22.65 \%$, respectively. Both survival rates

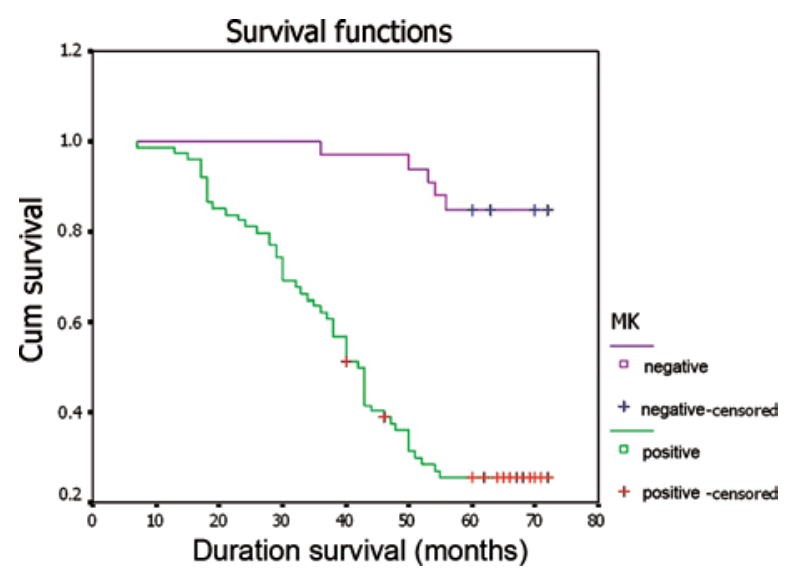

Figure 3. Survival curves of gastric cancer patients with different expression of MK.

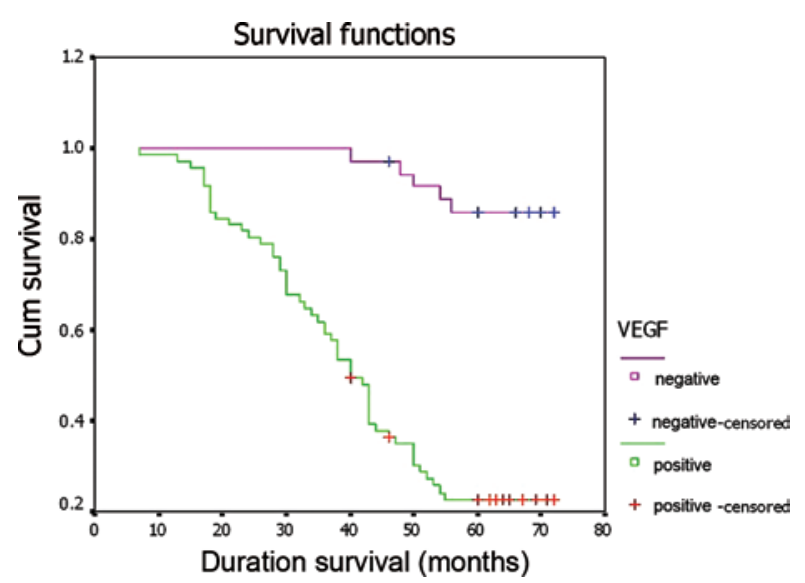

Figure 4. Survival curves of gastric cancer patients with different expression of VEGF.

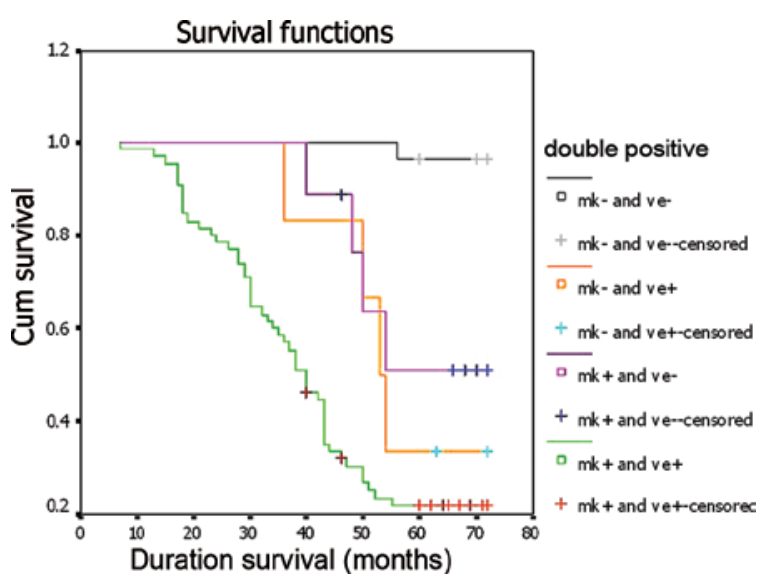

Figure 5. Survival curves of gastric cancer patients with different expression of MK and VEGF.

were significantly lower than those of patients with MK- or VEGF-negative tumors. Analysis using Cox's proportional hazards model with forward stepwise regression showed the independence of tumor PTNM stage $(\mathrm{P}=0.003)$ and of MK with VEGF co-expression $(\mathrm{P}=0.003)$ as prognostic factors following radical gastrectomy (Figs. 3-5 and Table III). 
Table III. Cox multivariate analysis of parameters for the prognosis of gastric cancer patients.

\begin{tabular}{lccccc}
\hline Parameter & $\beta$ & Wald & P-value & RR & $\begin{array}{c}95 \% \text { confidence } \\
\text { interval }\end{array}$ \\
\hline $\begin{array}{l}\text { PTNM stage } \\
\text { (I+II vs. III+IV) }\end{array}$ & 1.879 & 8.572 & 0.003 & 6.546 & $1.861-23.024$ \\
$\begin{array}{l}\text { MK and VEGF expression } \\
\text { (double positive vs. others) }\end{array}$ & -1.191 & 9.129 & 0.003 & 0.304 & $0.140-0.658$ \\
\hline
\end{tabular}

PTNM, pathological tumor node metastasis; MK, midkine; VEGF, vascular endothelial growth factor; RR, relative risk. Others include $\mathrm{MK}^{+} \mathrm{VEGF}^{-}, \mathrm{MK}^{-} \mathrm{VEGF}^{+}$and $\mathrm{MK}^{-} \mathrm{VEGF}^{-}$.

\section{Discussion}

The gene for MK was cloned by Kadomatsu et al (13) in 1988. MK and pleiotrophin (PTN) belong to a family of heparinbinding growth factors (HBGFs). Under normal conditions, the MK level is high in the embryonic period and decreases after birth. In adults, no expression is found, except in the kidney and intestinal epithelium. Previous studies have shown that MK is highly and frequently expressed in a variety of cancer tissues, demonstrating that MK may be associated with tumorigenesis and tumor growth, invasion and metastasis (14-18). MK binds to complex membrane protein receptors, including protein tyrosine phosphatase $\zeta$ ( $\mathrm{PTP} \zeta$ ), low-density lipoprotein-related protein (LRP), anaplastic lymphoma kinase and syndecan $(19,20)$. Maeda et al (21) found that chondroitin sulfate proteoglycan binds to the heparin binding site of MK. In particular, the chondroitin sulfate proteoglycan PTP $\zeta$ showed marked affinity for MK. Shibata et al (22) confirmed that LRP, another component of the MK receptor, recognizes a variety of ligands, such as apolipoprotein E, and transports these ligands through endocytosis. LRP also activates $\alpha-2$ macroglobulin, transports MK into cells and transfers MK into the nucleus via nucleolin (a nuclear-cytoplasmic transfer protein).

Growth of solid tumors depends on angiogenesis. Cytokines, such as MK, VEGF, BFGF and IL-8, play a role in tumor neovascularization. MK regulates proliferation, enhances fibrinolysis, has anti-apoptotic activity and induces malignant transformation. In the study by Rha et al (23), 67\% of gastric cancer cells had high MK expression. Cells expressing MK are better able to form colonies on agar gel and to stimulate growth of vascular endothelial cells. Obata et al (24) showed that serum MK levels were significantly higher in patients with gastric cancer than in healthy individuals, and significantly higher in patients with advanced gastric cancer than in patients with early-stage disease, indicating that serum MK level (compared to histological staining) more directly reflects tumor stage. Huang et al (25) reported a positive correlation of MK level in gastric cancer tissues with clinical stage and lymph node metastasis, but not with tumor size and degree of tumor differentiation, suggesting that MK could be used as an effective biological indicator for early diagnosis of gastric cancer and prediction of prognosis.

VEGF promotes endothelial cell proliferation and angiogenesis, and increases vascular permeability to serum proteins. This provides a basis for migration of endothelial cells and metastasis of tumor cells (26). Numerous studies have confirmed that VEGF expression is high in a variety of malignant tumors. Maeda et al (27) detected VEGF expression in 129 gastric cancer samples by immunohistochemistry, and found a correlation between VEGF and tumor invasion and metastasis, suggesting that VEGF could be used as a molecular marker to evaluate the biological behavior and prognosis of gastric cancer.

In our immunohistochemical examination, MK and VEGF were highly expressed in gastric cancer tissues, but not in normal gastric mucosa. The difference in expression levels between these two tissues was significant $(\mathrm{P}=0.00)$. The MK and VEGF proteins were concentrated mainly in the cytoplasm (not in the nucleus) of gastric cancer cells. There was diffuse staining of tumor tissues and some marked differences in staining distribution between tissues, including enhanced expression at the gastric tissue edges, positive staining of fibrous tissue in the extracellular matrix of gastric cancer, and positive staining of small blood vessel endothelial cells (particularly in tissues with high vascular density). These results indicate that MK and VEGF act synergistically in stimulating tumor angiogenesis in gastric cancer. MK and VEGF expression was associated with tumor size, depth of invasion, lymph node metastasis and pathological stage, but not with the age and gender of patients. Expression was significantly higher in tumors with a diameter greater than $5 \mathrm{~cm}$, serosal invasion and lymph node involvement, and in patients with intermediate or advanced-stage disease $(\mathrm{P}<0.01)$. These results indicate that MK and VEGF act cooperatively to promote gastric cancer growth and cancer cell invasion and metastasis. We also found that the high MK and VEGF expression occurred mostly in patients with late-stage disease or poor prognosis, which is consistent with the results of Shimada et al (28). In this study, multivariate Cox analysis showed that coincident expression of MK and VEGF, but not MK or VEGF alone, may be used as an independent prognostic factor of gastric cancer, suggesting that MK and VEGF act synergistically in gastric cancer cell proliferation, invasion and metastasis. Further studies are required to determine the specific mechanism. These studies may lead to new cancer treatments, possibly using antisense oligonucleotides to target VEGF and MK genes and gene expression $(29,30)$.

\section{References}

1. Yang L: Incidence and mortality of gastric cancer in China. World J Gastroenterol 12: 17-20, 2006. 
2. Mandong BM, Manasseh AN, Tanko MN, Echejoh GO and Madaki AJ: Epidemiology of gastric cancer in Jos University Teaching Hospital, Jos: a 20 year review of cases. Niger J Med 19: 451-454, 2010

3. Takayama S, Wakasugi T, Funahashi $\mathrm{H}$ and Takeyama H: Strategies for gastric cancer in the modern era. World J Gastrointest Oncol 2: 335-341, 2010.

4. Wiwanitkit V: Helicobacter pylori screening to prevent gastric cancer: an economical analysis for a tropical developing country. Asian Pac J Cancer Prev 11: 571-572, 2010.

5. Bornschein J, Kandulski A, Selgrad M and Malfertheiner P: From gastric inflammation to gastric cancer. Dig Dis 28: 609-614, 2010.

6. Windgassen EB, Funtowicz L, Lunsford TN, Harris LA and Mulvagh SL: C-reactive protein and high-sensitivity C-reactive protein: an update for clinicians. Postgrad Med 123: 114-119, 2011.

7. Oshima Y, Yajima S, Yamazaki K, Matsushita K, Tagawa M and Shimada H: Angiogenesis-related factors are molecular targets for diagnosis and treatment of patients with esophageal carcinoma. Ann Thorac Cardiovasc Surg 16: 389-393, 2010.

8. Lucas S, Reindl T, Henze G, Kurtz A, Sakuma S and Driever PH: Increased midkine serum levels in pediatric embryonal tumor patients. J Pediatr Hematol Oncol 31: 713-717, 2009

9. Dai LC: Midkine translocated to nucleoli and involved in carcinogenesis. World J Gastroenterol 15: 412-416, 2009.

10. Buchler P, Reber HA, Buchler M, et al: Hypoxia-inducible factor 1 regulates vascular endothelial growth factor expression in human pancreatic cancer. Pancreas 26: 56-64, 2003.

11. Cabuk D, Basaran G, Celikel C, et al: Vascular endothelial growth factor, hypoxia-inducible factor 1 alpha and CD34 expressions in early-stage gastric tumors: relationship with pathological factors and prognostic impact on survival. Oncology 72: 111-117, 2007.

12. Vidal O, Metges JP, Elizalde I, et al: High preoperative serum vascular endothelial growth factor levels predict poor clinical outcome after curative resection of gastric cancer. Br J Surg 96: $1443-1451,2009$

13. Kadomatsu K, Tomomura M and Muramatsu T: cDNA cloning and sequencing of a new gene intensely expressed in early differentiation stages of embryonal carcinoma cells and in midgestation period of mouse embryogenesis. Biochem Biophys Res Commun 151: 1312-1318, 1988.

14. Kurtz A, Schulte AM and Wellstein A: Pleiotrophin and midkine in normal development and tumor biology. Crit Rev Oncog 6: 151-177, 1995.

15. Choudhuri R, Zhang HT, Donnini S, Ziche M and Bicknell R: An angiogenic role for the neurokines midkine and pleiotrophin in tumorigenesis. Cancer Res 57: 1814-1819, 1997.
16. Kadomatsu $\mathrm{K}$ and Muramatsu T: Midkine and pleiotrophin in neural development and cancer. Cancer Lett 204: 127-143, 2004

17. Friedrich $\mathrm{C}$, Holtkamp $\mathrm{N}$, Cinatl $\mathrm{J} \mathrm{Jr}$, et al: Overexpression of Midkine in malignant peripheral nerve sheath tumor cells inhibits apoptosis and increases angiogenic potency. Int J Oncol 27: 1433-1440, 2005.

18. Nobata S, Shinozawa $\mathrm{T}$ and Sakanishi A: Truncated midkine induces transformation of cultured cells and short latency of tumorigenesis in nude mice. Cancer Lett 219: 83-89, 2005.

19. Chen S, Bu G, Takei Y, et al: Midkine and LDL-receptor-related protein 1 contribute to the anchorage-independent cell growth of cancer cells. J Cell Sci 120: 4009-4015, 2007.

20. Stoica GE, Kuo A, Powers C, et al: Midkine binds to anaplastic lymphoma kinase (ALK) and acts as a growth factor for different cell types. J Biol Chem 277: 35990-35998, 2002.

21. Maeda N, Ichihara-Tanaka K, Kimura T, Kadomatsu K, Muramatsu T and Noda M: A receptor-like protein-tyrosine phosphatase PTPzeta/RPTPbeta binds a heparin-binding growth factor midkine. Involvement of arginine 78 of midkine in the high affinity binding to PTPzeta. J Biol Chem 274: 12474-12479, 1999.

22. Shibata Y, Muramatsu T, Hirai M, et al: Nuclear targeting by the growth factor midkine. Mol Cell Biol 22: 6788-6796, 2002.

23. Rha SY, Noh SH, Kwak HJ, et al: Comparison of biological phenotypes according to midkine expression in gastric cancer cells and their autocrine activities could be modulated by pentosan polysulfate. Cancer Lett 118: 37-46, 1997.

24. Obata Y, Kikuchi S, Lin Y, Yagyu K, Muramatsu T and Kumai H: Serum midkine concentrations and gastric cancer. Cancer Sci 96: 54-56, 2005.

25. Huang Y, Cao G, Wang H, Wang Q and Hou Y: The expression and location of midkine in gastric carcinomas of Chinese patients. Cell Mol Immunol 4: 135-140, 2007.

26. Tuder RM and Yun JH: Vascular endothelial growth factor of the lung: friend or foe. Curr Opin Pharmacol 8: 255-260, 2008.

27. Maeda K, Chung YS, Ogawa Y, et al: Prognostic value of vascular endothelial growth factor expression in gastric carcinoma. Cancer 77: 858-863, 1996.

28. Shimada H, Takeda A, Nabeya Y, et al: Clinical significance of serum vascular endothelial growth factor in esophageal squamous cell carcinoma. Cancer 92: 663-669, 2001.

29. Maehara H, Kaname T, Yanagi K, et al: Midkine as a novel target for antibody therapy in osteosarcoma. Biochem Biophys Res Commun 358: 757-762, 2007.

30. Kerzerho J, Adotevi O, Castelli FA, et al: The angiogenic growth factor and biomarker midkine is a tumor-shared antigen. J Immunol 185: 418-423, 2010. 